\title{
Speed of responding to mixed language signals ${ }^{1}$
}

\author{
E. C. DALRYMPLE-ALFORD AND ARWA AAMIRY \\ AMERICAN UNIVERSITY OF BEIRUT, LEBANON
}

In two experiments Ss were presented with 2-word unilingual and mixed language signals which indicated which of six keys were to be pressed. While the speed with which the correct response was made was unaffected by whether the signal was linguistically mixed or not, the language of the first word appeared to be critical.

The process of extracting information from a signal may be considered to involve the application of the appropriate set of decoding rules. Insofar as these rules are different for different languages, one would expect that the bilingual presented with a mixed language signal would be less proficient in extracting the information from it than from a unilingual signal because of having to switch from one system to the other. However, this does not seem to be the case. Kolers (1966) reports that Ss given enough time to read but not enough to study or memorize a passage did just as well on comprehension tests whether the passage was unilingual or mixed. This finding is somewhat surprising in view of reports that the bilingual's performance is impaired in other tasks, such as reading aloud and continuous verbal production, when switching between languages is required (Kolers, 1966; Macnamara, 1967). There is of course the possibility that the comprehension test used by Kolers was not sensitive enough to pick up the differences that exist. In the study reported here a different procedure was adopted to see whether information extraction is less efficient when the signals are linguistically mixed.

Ss were presented with two-word signals indicating which of six keys had to be pressed. The task was essentially a modification of Lambert's (1955) "automaticity" test of relative language proficiency. Here, however, half the signals were unilingual (English or Arabic) and half were linguistically mixed (ArabicEnglish, English-Arabic). A feature of this task is that since the same nonlinguistic response (key pressing) is made to all signals and these are well within the memory span, one may reasonably attribute differences in the speed of responding to differences in the ease with which information is extracted from the four types of signals.

\section{EXPERIMENT 1}

\section{Method}

Each signal consisted of two words typed one approximately $1.2 \mathrm{~cm}$ above the other. The first (i.e., top) word was either "left" or "right" or its Arabic equivalent. The second word in English or Arabic in- dicated the color of the key to be pressed, this being red, green, or yellow. Thus there were six possible responses (right-red key, left-red key, etc.) and four conditions (Arabic-Arabic, Arabic-English, EnglishEnglish and English-Arabic), making a total of 24 different signals. S was seated before a sloping response panel on which were mounted six telegraph keys. The colored keys were arranged in a semicircle about a "start" button attached to a microswitch in the following order (from left to right): green, yellow, red, red, yellow, green. Immediately beyond and above the response panel was a display unit (a modification of a simple tachistoscope) in which the cards with the signals were presented. When $S$ depressed the start button, the signal on the card was illuminated and a timer started. The display terminated and the timer stopped when S pressed any one of the six response keys. A panel of pilot lamps indicated which key had been pressed. Response times were measured to the nearest .01 sec. Two different orders of presentation were used. These orders were arrived at by putting together two random arrangements of the 24 different signals. The randomization was restricted in that not more than two sigmals from the same condition were allowed to follow one after the other. Half the Ss were presented with the resulting sequence of 48 signals which were then presented again but in reversed order. The remaining Ss had the reversed order. Thus all Ss had to respond to the 24 signals four times over.

The Ss were 20 Arabic-English bilingual university students.

\section{Resulis and Discussion}

In analyzing the results, the series of 96 signals was divided into first and second halves. Mean response times for the four conditions in each half were computed for each $\mathrm{S}$ after discarding erroneous responses of which there were approximately $4 \%$ distributed fairly equally among the four conditions. Analysis of variance carried out on these means revealed a significant difference between conditions $(F=4.87, \mathrm{df}=3 / 133, \mathrm{p}<.01)$, but not between halves

Table 1. Mean Response Times in sec

\begin{tabular}{lcr} 
First word in: & Arabic & English \\
\hline Second word in: & & 1.27 \\
Arabic & 1.18 & 1.23 \\
English & 1.17 & 1.27 \\
\hline
\end{tabular}


Table 2. Mean Response Times in sec

\begin{tabular}{lllll}
$\begin{array}{l}\text { First word: } \\
\text { Longuage }\end{array}$ & \multicolumn{2}{c}{ Arabic } & \multicolumn{2}{c}{ English } \\
\hline Content & Location & Color & Location & Color \\
\hline Second word in: & & & & \\
Arabic & 1.320 & 1.357 & 1.369 & 1.440 \\
English & 1.323 & 1.355 & 1.342 & 1.447 \\
\hline
\end{tabular}

$(F=1.88$, df $=1 / 133, p>.1)$ or the interaction between halves and conditions $(F<1)$. Table 1 sets out the means for the four conditions. Orthogonal comparisons (Winer, 1962) between these were tested for significance ( $d f=1 / 133$ in all cases). There was no significant difference between response times for mixed (Arabic-English, English-Arabic) and unilingual (English-English, Arabic-Arabic) signals ( $F<1$ ), nor between the response times for Arabic-Arabic and English-English signals $(F=2.65, p>.10)$. However, English-Arabic signals were responded to significantly more slowly than Arabic-English signals ( $F=11.45$, $\mathrm{p}<.001$ ).

While the data and their analysis support the conclusion that the $\mathrm{Ss}$ responded as quickly to mixed language signals as to unilingual ones, this conclusion fails to take account of what may prove a significant feature of the results-namely that the language of the first (or more correctly, top) word of the signal may determine the speed of responding to the signal, irrespective of whether the signal is unilingual or not. However, since the first word was always one of location (left/right) we may be dealing simply with the effect of differences in the speed of decoding the words for left, right, red, green, and yellow in the two languages. To get further clarification, a second experiment was run in which for half the trials for each of the four conditions the first word indicated location and for the other half indicated the color of the key.

\section{EXPERIMENT 2}

\section{Method}

The apparatus was the same as in Experiment 1 except for a reworking of the circuit to accommodate a more precise timer so that the measurements were to the nearest .002 sec. As before, two-word signals were used. The variables concerned may be specified in terms of the characteristics of the first word and the language of the second: (a) language of first word (Arabic/English), (b) "content" of first word(location/ color), and (c) language of second word (Arabic/English). The ordering of the 12 signals, used for each of the elght conditions that resulted, followed the same principles as in the previous experiment.
The Ss were 10 Arabic-English bilingual university students.

\section{Results and Discussion}

The mean error rate, approximately $5 \%$, was fairly constant for all conditions. After dividing the 96 trials into first and second halves and discarding the times for incorrect responses, mean response times were computed for each $S$ for the eight conditions in each half. Analysis of variance of these means revealed a significant difference between conditions $(F=2.84, \mathrm{df}=7 / 135, \mathrm{p}<.01)$ and between halves $(\mathrm{F}=$ $31.34, \mathrm{df}=1 / 135, \mathrm{p}<.001$ ), but the conditions by halves interaction was not significant $(F=1.003)$. The means for the eight conditions are set out in Table 2. Seven orthogonal comparisons between these means were tested for significance ( $d f=1 / 135$ in all cases) with the following outcomes (comparisons are numbered in brackets). (1) Signals with the first word in Arabic were responded to significantly faster than those with the first word in English ( $F=8.82, p<.005$ ). (2) Among the former, there was no significant difference ( $F=$ 1.42) between those with location (left/right) first and those with color first. (3) Signals with the first word in English were responded to significantly faster when the first word indicated location than when this word gave the color of the correct key $(F=9.13, p<.005)$. None of the remaining comparisons which were of main interest were significant ( $F<1$ in all cases). Response times for the Arabic-Arabic signals were not significantly faster than those for Arabic-English signals whether location (4) or color (5) was first, and the same was true for English-English and English-Arabic signals $(6,7)$. We see then that the results of this experiment substantiate those of the previous one. Ss respond just as fast to linguistically mixed as to unilingual signals having the same content when the first of the two words comprising the signals belong to the same language. We are unable as yet to suggest a satisfactory explanation of this.

\section{References}

KOLERS, P. A. Reading and talking bilingually. Amer. J. Psychol, 1966, 79, 357-376.

LAMBERT, W. E. Measurement of the linguistic dominance of bilinguals. J. abnorm. soc. Psychol, 1955, 50, 197-200.

MACNAMARA, J. The linguistic independence of bilinguals. $J$. verbal Learn. verbal Behav., 1967, in press.

WINER, B. J. Statistical principles in experimental design. New York: McGraw-Hill, 1962.

\section{Note}

1. This study was partly supported by a grant from the Arts and Sciences Research Committee of the American University of Beirut. We are grateful to Bushra Budayr for her assistance. 